\title{
Changes in neurotransmitter levels associated with the deficiency of some essential amino acids in the diet
}

\author{
BY JOSÉ L. VENERO, ANTONIO J. HERRERA, ALBERTO MACHADO \\ AND JOSEFINA CANO \\ Departamento de Bioquimica, Bromatología y Toxicologia, Universidad de Sevilla, C/ Prof. Garcia \\ González s/n, 41012-Sevilla, Spain
}

(Received 11 February 1991-Accepted 23 August 1991)

\begin{abstract}
The contents of dopamine (DA) and serotonin (5-HT) and their metabolites were measured in rat substantia nigra and corpus striatum following dietary changes, including restriction of protein content (low-protein diet; LPD) and the contents of several large neutral amino acids (isoleucine, leucine, methionine, phenylalanine, tryptophan and valine) for $25 \mathrm{~d}$. The LPD produced an increase in the concentration of tyrosine (TYR) in the two regions of the brain studied. This effect was also observed with all amino acid deficiencies studied except for valine in the substantia nigra, tryptophan in the striatum and phenylalanine in both regions. Likewise, the concentration of 5-hydroxyindolacetic acid (5HIAA), the main metabolite of 5-HT, increased in the substantia nigra but not in the striatum after LPD, as well as with all the amino acid deficiencies studied, with the exception of tryptophan deficiency. In this case there was a dramatic effect on all components of the serotoninergic system, with decreases in the concentration of tryptophan (TRP; precursor), 5-HT and 5-HIAA. This behaviour clearly shows an interrelationship between precursor (TRP) availability and 5-HT synthesis and metabolism. With valine deficiency, dopaminergic and serotoninergic systems demonstrated opposite effects in the substantia nigra and the corpus striatum, and the behaviour of the two monoamines was also opposite within each structure. The significance of these changes is discussed.
\end{abstract}

Substantia nigra: Striatum: Malnutrition: Monoamines

The amino acid composition of a protein, particularly the essential amino acid portion, is crucial for nutrition. Most dietary proteins, especially those derived from vegetable sources, are poor in some essential amino acids (Schoefield \& Booth, 1983). The intake of these essential amino acids is important in such functions as protein synthesis and growth (Stern et al. 1976; West \& Kemper, 1976). Several amino acids are also involved in biogenic amine biosynthesis, while others are themselves neurotransmitters.

The impact of protein malnutrition on the brain has been examined at different stages of life, primarily during development and maturation (Winick \& Rosso, 1973; Winick, 1989). The effect of loading or deficiency of essential amino acids such as tryptophan, phenylalanine or tyrosine has also been evaluated (Miller et al. 1976, 1977 a; During et al. 1989; Westerink \& De Vries, 1991) in an effort to determine the resulting impact on the synthesis and concentration of neurotransmitter substances (Fernstrom \& Wurtman, 1971; Miller et al. 1977 b). However, little attention has been focused on the comparative effect of different essential amino acid deficits and neurotransmitter synthesis in adults. Taking into account the real possibility of malnutrition at some time during the adult stage, we studied its effect on some aspects of brain function.

The present study was designed to determine the effect of a dietary deficiency in some of 
the essential amino acids on the level of biogenic amines acting as neurotransmitters in different areas of the central nervous system. The effect of the biogenic amines serotonin (5-HT) and dopamine (DA), their respective precursors tryptophan (TRP) and tyrosine (TYR), and their metabolites 5-hydroxyindolacetic acid (5-HIAA, 3,4-dihydroxyphenylacetic acid (DOPAC), 3-methyoxytyramine (3-MT) and homovanillic acid (HVA) was investigated, because it is known that these neurotransmitters are influenced by different amino acids, especially tryptophan and phenylalanine, as precursors for indolamines and catecholamines respectively. At the same time, they are transported across the blood-brain barrier by the large neutral amino acid transport system, so they must compete with valine, leucine, isoleucine, tyrosine and methionine for access to the carrier-binding site (Pardridge, 1983). For these reasons these amino acids were selected. The two regions selected for the study were the corpus striatum and the substantia nigra of the rat because they are extremely rich in neurotransmitter content, allowing measurement of changes in their concentrations, and because in human patients with various neurological disorders changes in biogenic amine levels have been described in these areas. Thus, Parkinson's disease is associated with a degeneration of dopaminergic cell bodies of the pars compact of the substantia nigra (Hirsch et al. 1988) and a decrease in DA concentration in the striatum (Marsden, 1982). The nigro-striatal system has also been reported to be involved in other neurological disorders. For instance, Huntington's disease is associated with severe degeneration and cell loss in the corpus striatum, where efferent neurons of all the striatal neurotransmitters are affected (Enna et al. 1977; Penney \& Young, 1982; Scheel-Krüger, 1986). Likewise, there is degeneration of the melanin-pigmented neurons in the substantia nigra in Alzheimer's disease (Mann et al. 1982).

The effects were studied in adult rats: (1) fed on the standard control diet (CD); (2) fed on a low-protein diet supplemented with all essential amino acids (LPD; this diet having all the nutritional requirements recommended by National Academy of Sciences (1978)); (3) fed on a low-protein diet-supplemented with all essential amino acids except one.

The findings support the hypothesis that a deficiency in some of these amino acids induces changes in biogenic amine levels. In some cases, there is a decrease in the concentration of dopamine or serotonin that could exacerbate an incipient pathological disease related to these levels.

\section{EXPERIMENTAL METHODS}

\section{Animals and treatment}

Thirty-two male Wistar rats weighing 150-200 $\mathrm{g}$ and born in our laboratory were used. Animals were housed in eight groups (four rats per cage) under a $12 \mathrm{~h}$ light-dark cycle. They were fed on a standard commercial diet (Panlab) until administration of the experimental diets: CD, LPD and LPD without one of the essential amino acids to be tested. The composition of these diets was as follows.

$C D$. This contained $(\mathrm{g} / \mathrm{kg}) 180$ lactalbumin, 220 sucrose, 440 dextrin, and 110 fat, with the remainder of the diet composed of vitamins and minerals (rat diet corrector vitamin and minerals, Incoler-1; Incovesa, Leon). The total amino acid composition is shown in Table 1.

$L P D$. This was an isoenergetic diet containing $(\mathrm{g} / \mathrm{kg}) 5$ lactalbumin, 270 sucrose, 560 dextrin, and 110 fat, as well as lysine $14 \cdot 6$, threonine $5 \cdot 0$, arginine $14 \cdot 9$, histidine $5 \cdot 4$, methionine $4 \cdot 8$, isoleucine 5 , leucine $7 \cdot 5$, phenylalanine $8 \cdot 0$, tryptophan $2 \cdot 6$, valine $6 \cdot 0$, with the remainder of the diet composed of vitamins and minerals. Details of the amino acid composition of LPD are shown in Table 1. 
Table 1. Amino acid composition $(\mathrm{g} / \mathrm{kg})$ of control diet $(C D)$ and low-protein diet $(L P D)$

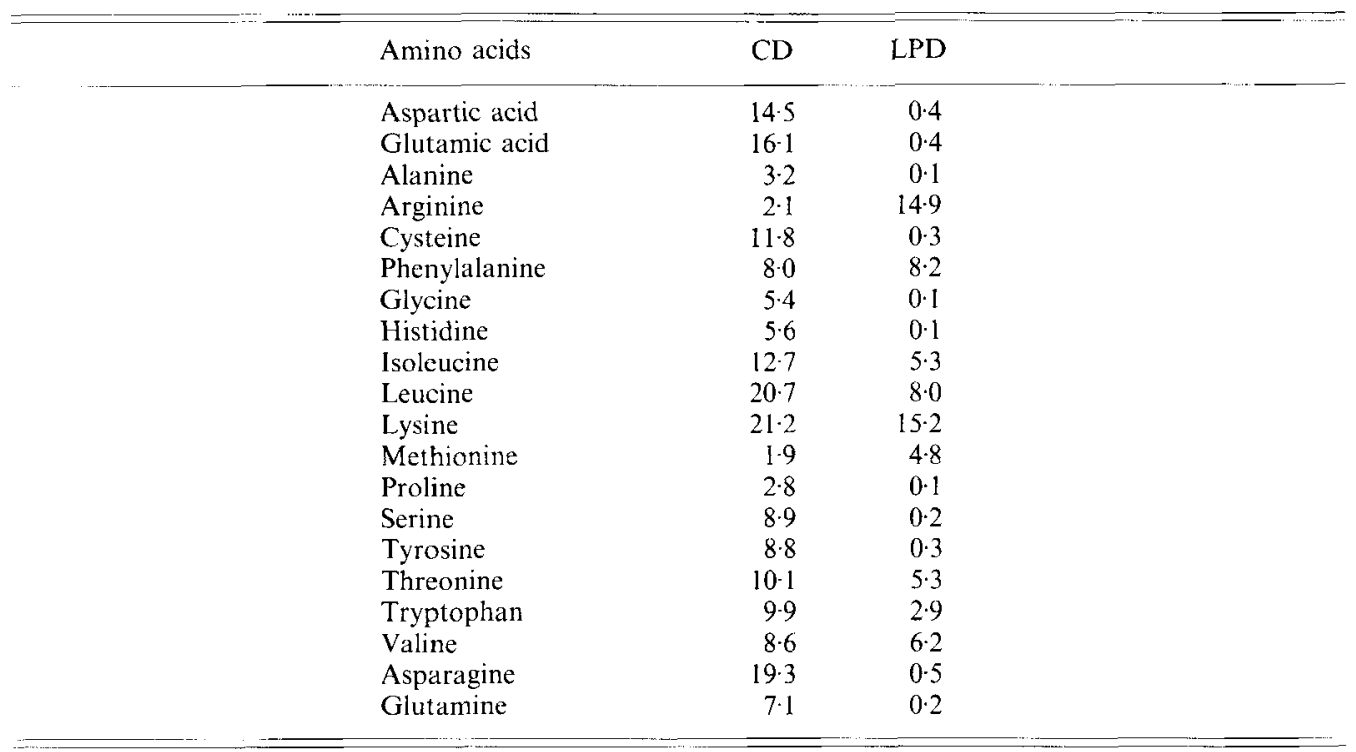

\section{Experimental diets}

Six different experimental diets were prepared. The composition of each diet was identical to LPD except for the omission of methionine, isoleucine, leucine, phenylalanine, tryptophan or valine.

Throughout the study all rats were given food and water ad lib. The diets were fed for $25 \mathrm{~d}$ before each experiment. The food intake was recorded daily and corrected for spillage.

All animals were decapitated between 10.00 and 11.00 hours and the whole brain was quickly removed. The mesencephalon was divided into two parts, with an incision from the ventral side at the caudal border eminence running perpendicular to the long axis of the mesencephalon. The two substantia nigras were then easily identified, and dissected free of the surrounding tissue, including the ventral tegmental area (A 10). The resulting portion was frozen in liquid nitrogen, as was a similarly-prepared portion of the striatum. The total time-period required for isolation of each portion was less than $3 \mathrm{~min}$.

\section{Measurement of biogenic amines}

Analyses were performed by means of a high-performance liquid chromatograph equipped with a Kontron 420 pump with electrochemical detection (Bioanalytical System, LC-4B). Measurement of the biogenic amines and their metabolites was performed according to methods described in detail elsewhere (Venero et al. 1989). Typical experimental chromatograms are shown in Fig. 1. Chromatograms were recorded on a Merck-Hitachi integrator (model D-2000). Accurate integrating options were required for integrating TYR in both substantia nigra and striatum, and DOPAC in the striatum. In order to obtain an adequate integration for both metabolites, a tail option was chosen for integrating TYR, and a perpendicular drop for DOPAC. 

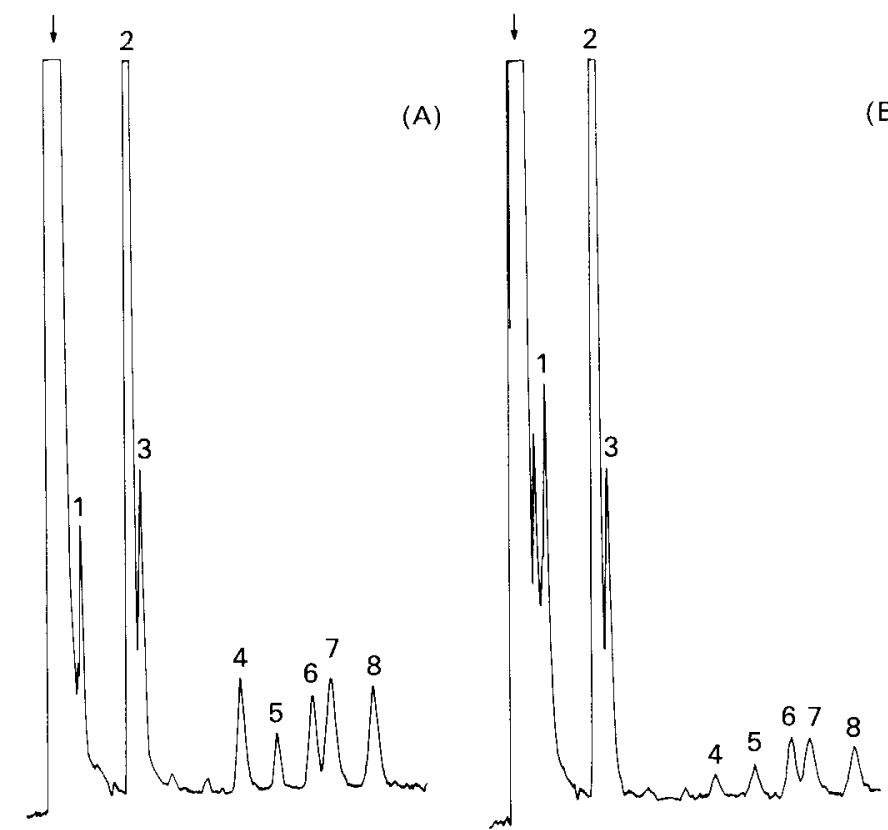

Fig. 1. Chromatograms of dopamine (DA), serotonin (5-HT) and their metabolites from: (A) rat striatal tissue after $25 \mathrm{~d}$ on a low-protein diet; (B) rat striatal tissue after $25 \mathrm{~d}$ on a tryptophan-deficient diet. $\downarrow$, injection peak; 1, tyrosine; 2, dopamine; 3, 3,4-dihydroxyphenylacetic acid; 4, 5-hydroxy-3-indolacetic acid; 5, 3-methoxytyramine; 6, 5-HT; 7, homovanillic acid; 8, tryptophan. For details of diets, see pp. 4I0-4lI and Table 1.

\section{Statistical analysis}

Biogenic amine contents from animals fed on CD and LPD were compared using Student's $t$ test. Data from LPD and the different amino acid-deficient diets were analysed by means of one-way analysis of variance (ANOVA). Observed mean differences were evaluated using the Schefee test (parametric test). Food intake and body-weight loss were compared using Student's $t$ test.

\section{RESULTS}

Food intake and body-weight loss

There were no significant differences in food intake (g/animal per $\mathrm{d}$ ) between CD and LPD and the different experimental diets: CD 16.7 (SE 1.4), LPD and experimental diets 15.2 (SE 1.4). There were no differences in body-weight loss (\%): CD 10.6 (SE 0.9), LPD and experimental diets $13 \cdot 2$ (SE 2.1). The fact that all treatments led to a certain degree of bodyweight loss could be attributed to the low palatability of the diets. This is supported by the finding that the highest loss rate was recorded during the first week of the treatments. However, from day 8 to 25 (before death) the respective body-weights remained almost unchanged.

\section{Effect of LPD on neurotransmitter levels}

There were no significant changes in the serotoninergic system, except for 5-HIAA which increased in the substantia nigra with LPD when compared with CD $(38.0 \% ; P<0.01$; Table 2). Moreover, little change was found in the concentration of 5-HT in the two regions after LPD, but there was an increase in the substantia nigra and a decrease in the striatum. Within the dopaminergic system, an increase in the concentration of TYR after LPD was seen in both structures $(42.2 \%$ in the substantia nigra, $25.4 \%$ in the striatum; $P<0.01$; Tables 3 and 5). 
Table 2. Effects of various diets on the concentration (ng/g wet tissue) of serotonin (5$H T)$ and its metabolites in the substantia nigra of rats

(The combined SEM and df for each compound were: TRP SEM 54.2, df 21 ; 5-HT SEM 48.7, df 21 ; 5HIAA SEM $18 \cdot 7$, df 23 )

\begin{tabular}{|c|c|c|c|}
\hline Diet $\dagger$ & TRP & $5-\mathrm{HT}$ & 5-HIAA \\
\hline $\mathrm{CD}$ & 3386.4 & $2018 \cdot 4$ & $452 \cdot 4$ \\
\hline LPD & 3518.9 & 2164.9 & $624 \cdot 5 \ddagger$ \\
\hline \multicolumn{4}{|l|}{ LPD deficient in: } \\
\hline Isoleucine & $3344 \cdot 1$ & $2001 \cdot 8$ & $582 \cdot 4$ \\
\hline Leucine & $3727 \cdot 9$ & $1811 \cdot 5^{* *}$ & $640 \cdot 8$ \\
\hline Methionine & $3312 \cdot 8$ & $1703 \cdot 7^{* *}$ & $627 \cdot 7$ \\
\hline Phenylalanine & $3344 \cdot 3$ & $1819 \cdot 5^{*}$ & $642 \cdot 3$ \\
\hline Tryptophan & $1731 \cdot 9^{* *}$ & $1051 \cdot 4^{* *}$ & $250 \cdot 1^{* *}$ \\
\hline Valine & $3526 \cdot 9$ & $1714 \cdot 5^{* *}$ & $575 \cdot 7$ \\
\hline
\end{tabular}

CD, control diet; LPD, low-protein diet; TRP, tryptophan; 5-HIAA, 5-hydroxyindolacetic acid.

Mean values for amino acid-deficient diets were significantly different from those for LPD (one-way analysis of variance followed by Schefee test): ${ }^{*} P<0.05, * * P<0.01$.

$\dagger$ For details of diets, see pp. $410-411$ and Table 1 .

Mean value for LPD was significantly different from that for CD (Student's $t$ test): $\ddagger P<0.01$.

Table 3. Effects of various diets on the concentration $(\mathrm{ng} / \mathrm{g}$ wet tissue) of dopamine (DA) and its metabolites in the substantia nigra of rats

(The combined SEM and $\mathrm{df}$ for each compound were: TYR SEM $212 \cdot 1$, df 21 ; DA SEM $18 \cdot 1$, df 21 ; DOPAC SEM $3 \cdot 6$, df $21 ; 3-M T$ SEM $2 \cdot 6$, df 21 ; HVA SEM $4 \cdot 6$, df 21 )

\begin{tabular}{|c|c|c|c|c|c|}
\hline $\operatorname{Diet}^{\dagger}$ & TYR & DA & DOPAC & 3-MT & HVA \\
\hline $\mathrm{CD}$ & $6083 \cdot 2$ & $710 \cdot 5$ & $102 \cdot 2$ & $51 \cdot 1$ & $130 \cdot 5$ \\
\hline LPD & $8655.6 \$$ & $769 \cdot 7$ & $98 \cdot 0$ & $47 \cdot 8$ & $138 \cdot 7$ \\
\hline \multicolumn{6}{|l|}{ LPD deficient in: } \\
\hline Isoleucine & 8053.0 & $572 \cdot 7 * *$ & 111.7 & $56 \cdot 9$ & $109 \cdot 8^{*}$ \\
\hline Leucine & $10803 \cdot 6^{* *}$ & $649 \cdot 0^{*}$ & $139 \cdot 8^{* *}$ & $50 \cdot 0$ & 131.7 \\
\hline Methionine & 8767.4 & $762 \cdot 7$ & 115.7 & $51 \cdot 5$ & $124 \cdot 3$ \\
\hline Phenylalanine & $6813 \cdot 5^{* *}$ & $550 \cdot 2^{* *}$ & $80 \cdot 8$ & $50-9$ & $119 \cdot 1$ \\
\hline Tryptophan & $7905 \cdot 2$ & $584 \cdot 9 * *$ & $110 \cdot 7$ & $53 \cdot 7$ & $131 \cdot 5$ \\
\hline Valine & $7108^{*}$ & $838 \cdot 8$ & $139 \cdot 5^{* *}$ & $51 \cdot 6$ & $178.9 * *$ \\
\hline
\end{tabular}

CD, control diet; LPD, low-protein diet; TYR, tyrosine; DOPAC, 3,4-dihydroxyphenylacetic acid; 3-MT, 3methoxytyramine; HVA, homovanillic acid.

Mean values for amino acid-deficient diets were significantly different from those for LPD (one-way analysis of variance followed by Schefee test): ${ }^{*} P<0.05,{ }^{* *} P<0.01$.

$\dagger$ For details of diets, see pp. $410-411$ and Table 1 .

Mean value for LPD was significantly different from that for CD (Student's $t$ test): $\ddagger P<0 \cdot 01$.

\section{Substantia nigra}

Isoleucine deficit. This deficit had no effect on the serotoninergic system. Within the dopaminergic system this deficit was associated with a decrease in the concentrations of DA $(-25.6 \% ; P<0.01)$ and HVA $(-21.0 \% ; P<0.05)$ compared with that of LPD (Table 3).

Leucine deficit. Compared with LPD this deficit induced a decrease in the concentration of 5 -HT $(-16.3 \%, P<0.01$; Table 2$)$. The 5-HIAA: 5 -HT ratio was also increased compared with that obtained with LPD $(20 \cdot 6 \%)$. With respect to the dopaminergic system, 
there was an increase in the concentrations of TYR and DOPAC when compared with those obtained with LPD (24.8 and $41.8 \%$ for TYR and DOPAC respectively; $P<0.01$; Table 3), whereas DA concentrations decreased $(-15.6 \%, P<0.05$; Table 3$)$. The efflux of DA, quantified as DOPAC:DA ratio (Altar et al. 1987), was also increased compared with that obtained with LPD $(69 \cdot 2 \%)$.

Methionine deficit. This deficit caused a decrease in the concentration of 5-HT when compared with that found with LPD $(-21.3 \% ; P<0.01$; Table 2$)$. However, the efflux of 5-HT, measured as 5-HIAA:5-HT ratio, increased with respect to LPD $(27 \cdot 5 \%)$. No statistical change was detected in the dopaminergic system after this deficit.

Phenylalanine deficit. Deficit of this amino acid caused a decrease in the concentration of 5 -HT when compared with that obtained with LPD $(-15.9 \% ; P<0.05$; Table 2$)$. The turnover of 5-HT, measured as 5-HIAA : 5-HT ratio, increased compared with that found with LPD $(20.6 \%)$. Within the dopaminergic system there was a significant decrease in the concentrations of TYR $(-21.2 \% ; P<0.01)$ and DA $(-28.4 \% ; P<0.01)$ compared with these obtained with LPD (Table 3 ).

Tryptophan deficit. This deficit had a dramatic effect on all components of the serotoninergic system, showing a relationship between precursor availability and 5-HT synthesis. Thus, there was a decrease in the concentrations of TRP $(-50 \cdot 7 \%), 5-\mathrm{HT}$ $(-51.4 \%)$ and 5-HIAA $(-59.9 \% ; P<0.01)$ compared with those obtained with LPD (Table 2). With respect to the dopaminergic system this deficit decreased the concentration of DA with respect to LPD $(-24.0 \% ; P<0.01$; Table 3$)$; the DOPAC:DA ratio was increased compared with that obtained with LPD (46.1\%).

Valine deficit. This deficit caused a decrease in the concentration of 5-HT when compared with that for LPD $(-20.8 \%, P<0.01)$ (Table 2$)$. Within the dopaminergic system the concentrations of DOPAC and HVA were increased compared with those obtained with LPD $(42.3 \%$ for DOPAC and $28.9 \%$ for HVA; $P<0.01)$, and that of TYR decreased $(-17.8 \% ; P<0.05$; Table 3$)$. Moreover, the DOPAC:DA ratio was increased compared with that found with LPD $(30 \cdot 7 \%)$.

\section{Striatum}

Isoleucine deficit. This deficit had no effect on the serotoninergic system. Within the dopaminergic system, only HVA concentration decreased compared with that obtained with LPD $(-32.9 \% ; P<0.01$; Table 5).

Leucine deficit. Deficit of leucine caused a decrease in the concentration of 5-HT compared with that obtained with LPD $(-24.8 \%, P<0.05$; Table 4$)$. In the dopaminergic system there was an increase in the concentrations of TYR and DOPAC compared with those found with LPD $(83.4 \%$ for TYR and $42.7 \%$ for DOPAC; $P<0.01)$, and a decrease in HVA $(-10.8 \% ; P<0.01$; Table 5$)$; the DOPAC:DA ratio was increased compared with that with LPD $(31 \cdot 2 \%)$.

Methionine deficit. Deficit of methionine caused only a decrease in the TRP concentration compared with that obtained with LPD $(-25.6 \%, P<0.01)$ in the serotoninergic system (Table 4). In the dopaminergic system there was a decrease in the concentrations of TYR $(-27.6 \%, P<0.05)$ and HVA $(-22.7 \%, P<0.01)$ when compared with those for LPD (Table 5).

Phenylalanine deficit. This deficit only had an effect on the dopaminergic system. Thus, there was a decrease in the concentrations of TYR $(-33.7 \%, P<0.01)$ and HVA compared with those for LPD $(-21.3 \%, P<0.01$; Table 5). These results were accompanied by an increased DOPAC:DA ratio with respect to LPD (25.0\%).

Tryptophan deficit. This deficit, as for the substantia nigra, had a marked influence on all the components of the serotoninergic system. Thus, there was a decrease in the 
Table 4. Effects of various diets on the concentration ( $\mathrm{ng} / \mathrm{g}$ wet tissue) of serotonin (5-HT) and its metabolites in the striatum of rats

(The combined SEM and df for each compound were: TRP SEM 134.6, df $21 ; 5$-HT SEM 39.7, df 22; 5-HIAA SEM $10 \cdot 5$, df 24)

\begin{tabular}{|c|c|c|c|}
\hline Diet $†$ & TRP & 5-HT & 5-HIAA \\
\hline $\mathrm{CD}$ & $4623 \cdot 7$ & $1056 \cdot 4$ & $281 \cdot 8$ \\
\hline LPD & $4677 \cdot 3$ & 9106 & $301 \cdot 6$ \\
\hline \multicolumn{4}{|l|}{ LPD deficient in: } \\
\hline Isoleucine & $3994 \cdot 4$ & $1131 \cdot 2$ & $315 \cdot 2$ \\
\hline Leucine & $4961 \cdot 1$ & $684 \cdot 3^{*}$ & $256 \cdot 9$ \\
\hline Methionine & $3476 \cdot 6^{* *}$ & $1021 \cdot 5$ & $295 \cdot 2$ \\
\hline Phenylalanine & 4437.9 & $843 \cdot 5$ & $288 \cdot 6$ \\
\hline Tryptophan & $2440 \cdot 6^{* *}$ & $566 \cdot 3^{* *}$ & $135 \cdot 9^{* *}$ \\
\hline Valine & $4895 \cdot 7$ & $1169 \cdot 2^{*}$ & $290 \cdot 2$ \\
\hline
\end{tabular}

CD, control diet; LPD, low-protein diet; TRP, tryptophan; 5-HIAA, 5-hydroxyindolacetic acid.

Mean values for amino acid-deficient diets were significantly different from those for LPD (one-way analysis of variance followed by Schefee test): ${ }^{*} P<0-05 ;{ }^{*} P<0 \cdot 01$.

$\dagger$ For details of diets, see pp. $410-411$ and Table 1 .

Table 5. Effects of various diets on the concentration $(\mathrm{ng} / \mathrm{g}$ wet tissue) of dopamine $(D A)$ and its metabolites in the striatum of rats

(The combined SEM and df for each compound were: TYR SEM 376.4, df 21 ; DA SEM 396.7, cf 24 ; DOPAC SEM $83 \cdot 2$, df $23 ; 3$-MT SEM $22 \cdot 8$, df 24 ; HVA SEM $25 \cdot 8$, df 23 )

\begin{tabular}{|c|c|c|c|c|c|}
\hline $\operatorname{Diet}{ }^{\dagger}$ & TYR & DA & DOPAC & 3-MT & HVA \\
\hline $\mathrm{CD}$ & $5950 \cdot 8$ & $12220 \cdot 4$ & 1903.4 & $547 \cdot 1$ & $889 \cdot 7$ \\
\hline LPD & $7466 \cdot 7 \ddagger$ & $12599 \cdot 9$ & $1989 \cdot 3$ & 549.9 & $882 \cdot 0$ \\
\hline \multicolumn{6}{|l|}{ LPD deficient in: } \\
\hline Isoleucine & $6646 \cdot 0$ & $10548 \cdot 2$ & $2170 \cdot 4$ & 505.9 & $591 \cdot 2 * *$ \\
\hline Leucine & $13693 \cdot 0^{* *}$ & $13606 \cdot 6$ & $2839 \cdot 8 * *$ & $640 \cdot 1$ & $7860^{* *}$ \\
\hline Methionine & $5402 \cdot 3$ & $11208 \cdot 6$ & $2117 \cdot 7$ & $649 \cdot 9$ & $681 \cdot 8^{* *}$ \\
\hline Phenylalanine & $4944 \cdot 6^{* * *}$ & 10844.9 & $2112 \cdot 5$ & $518 \cdot 1$ & $694 \cdot 3^{* *}$ \\
\hline Tryptophan & $6682 \cdot 4$ & 122810 & $1987 \cdot 2$ & $582 \cdot 7$ & $586 \cdot 0^{* *}$ \\
\hline Valine & $7502 \cdot i$ & $83990^{* *}$ & $1482 \cdot 8^{* *}$ & $412 \cdot 2^{* *}$ & $414 \cdot 5^{* *}$ \\
\hline
\end{tabular}

CD, control diet; LPD, low-protein diet; TYR, tyrosine; DOPAC, 3,4-dehydroxyphenylacetic acid; 3-MT, 3methoxytyramine; HVA, homovanillic acid.

Mean values for amino acid-deficient diets were significantly different from those for LPD (one-way analysis of variance followed by Schefee test): ${ }^{*} P<0 \cdot 05, * * P<0 \cdot 01$.

$\dagger$ For details of diets, see pp. $410-411$ and Table 1 .

Mean value for LPD was significantly different from that for CD (Student's $t$ test): $\ddagger P<0.01$.

concentration of TRP $(-47.8 \%), 5$-HT $(-55.1 \%)$ and 5 -HIAA $(-55.1 \% ; P<0.01$; Table 4). In contrast to the serotoninergic system, in the dompaminergic system there was only a decrease in the HVA concentration, compared with that obtained with LPD $(-33.5 \% ; P<0.01)$ (Table 5).

Valine deficit. Within the serotoninergic system, only an increase in 5-HT concentration compared with LPD was detected $(28.3 \% ; P<0.05$; Table 4$)$. In the dopaminergic system the effect was opposite to that seen in the substantia nigra following this deficit. Thus, except TYR, the concentrations of all components of this neurotransmitter system were 
decreased with respect to LPD: DA $-33.3 \%$, DOPAC $-25.4 \%, 3-\mathrm{MT}-25.0 \%$ and HVA $-53.0 \%(P<0.01$; Table 5$)$.

\section{DISCUSSION}

We have studied the changes in neurotransmitter content in adult animals fed on diets deficient in some essential amino acids for a short period of time ( $25 \mathrm{~d})$. This situation may be found in some pathological conditions and may be important in older people. Thus, with age there is an increase in the appearance of some pathological diseases such as Parkinson's, related to the concentration of various neurotransmitters in different areas of the central nervous system. If the state of malnutrition affects the neurotransmitter content it could mean an enhancement of the risk of this kind of disease.

The low-protein diet supplemented with all essential amino acids met all the recommended nutritional requirements (National Academy of Sciences, 1978); this is confirmed by the low level of effect found on the concentrations of the biogenic amines in both regions studied. However, caution must be exercised because some differences between CD and LPD were detected These include a statistical increase in the concentration of TYR in both regions, and of 5-HIAA in the substantia nigra. Other changes, although not statistically significant, were those found for 5-HT in both regions, with an increase in one and a decrease in the other. Of the changes found, only that of TYR appeared to be related to the low dietary protein content, since similar effects were found in the two regions studied which was contrary to the changes seen in the serotoninergic system. With the available information it is difficult to explain the differences in TYR concentration between CD and LPD. Taking into account that phenylalanine does not act as a precursor of TYR in the central nervous system (there is no phenylalanine hydroxylase $(E C 1.14 .16 .1)$ activity in the brain), the increase seen in TYR with LPD could be related to a major efflux of TYR to the brain via the large neutral amino acid transport system. For this to occur, nitrogen from essential amino acids would be necessary to synthesize non-essential amino acids, including TYR, or it is possible that phenylalanine is converted enzymically to tyrosine by phenylalanine hydroxylase, in peripheral tissues, or both. The latter possibility seems to be plausible because of the similarity in the phenylalanine content in CD and LPD (see Table 1).

\section{Effect of some essential amino acid deficiencies in the serotoninergic system}

Dietary deficiency affected 5-HT metabolism in both regions studied, being more obvious in the substantia nigra. Notable was the increase in the concentration of 5-HIAA in the substantia nigra. A similar effect was found in this structure in isoleucine-deficient rats, and intensified in leucine, methionine and phenylalanine deficiencies. Moreover, with the latter treatments the increase in 5-HIAA was also accompanied by a significant decrease in the concentration of 5-HT with respect to CD and LPD. The different regional effects on 5-HT metabolism associated with the different amino acid deficiencies could be due to the different origins of the 5-HT. The 5-HT in the substantia nigra comes principally from the neuronal cell bodies and terminals (Fuxe, 1965; Ungerstedt, 1971; Olsen \& Seiger, 1972), whereas in the striatum it comes only from raphe nuclei terminals (Bobillier et al. 1976; Van der Kooi, 1979). The decrease in 5-HT content could be due to an acceleration of 5-HT turnover such as that described in serotoninergic nerve terminals injured by 5,7dihydroxytryptamine (Stachowiak et al. 1986). In the latter condition the levels of 5-HT and 5-HIAA were reduced in the hippocampus and septum after the lesion. However, as the magnitude of this decrease was much lower for 5-HIAA content, the 5-HIAA:5-HT ratio was increased. As 5-HIAA is the major metabolite of 5-HT in the brain, an increase 
in the 5-HIAA : 5-HT ratio may indicate an accelerated turnover of 5-HT. This possibility is supported by a previous report that formation of $5-\left[{ }^{3} \mathrm{H}\right] \mathrm{HIAA}$ from $\left[{ }^{3} \mathrm{H}\right] \mathrm{TRP}$ in the medulla and spinal cord increased after 5,7-dihydroxytryptamine administration (Gerson \& Baldessarini, 1974). In the present study a similar effect was also found in the striatum when there was a leucine or valine deficiency. These findings suggest that in rats fed on LPD brain protection decreases somewhat, especially in the substantia nigra. The lack of protection was more significant when there was a deficiency in leucine, methionine or phenylalanine. These findings are similar to those obtained by inducing different kinds of damage (Gerson \& Baldessarini, 1974; Stachowiak et al. 1986). If we relate the magnitude of the damage to the increase in the 5-HIAA:5-HT ratio, it is highest with the methioninedeficient diet. This fact may be related to the role of methionine, since this is a sulphurcontaining essential amino acid which is involved in the synthesis of cysteine. Cysteine is a fundamental component of the peptide glutathione. This peptide constitutes the major non-protein sulphurated component of all organisms and is involved in several important processes including detoxification (Sies et al. 1983) and the oxidative metabolism of central catecholamines (Hothersall et al. 1982). Thus, glutathione is fundamental in maintaining the cellular redox state (Reed et al. 1983). A deficit of methionine produces a marked decrease in hepatic glutathione concentration and should affect brain glutathione concentration, although to a lesser extent. This effect may also be the result of a decrease in protection against oxidative damage as has been reported in a vitamin E-deficient diet (A. Castaño, J. Cano \& A. Machado, unpublished results). This may be important since little is known about the mechanisms behind the degenerative process leading, for example, to Parkinson's disease, but it has been suggested that toxic species evolved from the catabolism of catechols may be involved in the pathogenesis (Cohen, 1983; Fornstedt et al. 1990).

A deficit of tryptophan affected 5-HT metabolism in both regions studied (5-HT and its metabolite 5-HIAA decreased). Since 5-HT in the brain is synthesized from the essential amino acid TRP, these results are in accordance with the fact that the rate of 5-HT synthesis in the brain depends on the availability of this precursor (Fernstrom \& Wurtman, 1971; Carlsson \& Lindqvist, 1978). Numerous experiments have demonstrated that an increase in brain TRP not only increases the rate of 5-HT synthesis but also increases the whole-brain concentration of 5-HIAA (Fernstrom \& Wurtman, 1971). Thus, a decrease in TRP concentration may be expected to produce a decrease in 5-HIAA. This finding may be important since, in some illnesses such as toxicity, TRP is administered as a scavenger (Gomez-Reino et al. 1983) without knowing the effect of this treatment on the free plasma TRP and subsequent TRP and 5-HT concentrations in the brain. Besides, little is known about whether radicals can oxidize TRP and how this would affect 5-HT concentration. However, from the amino acid composition of CD and LPD $(9.9 \mathrm{~g}$ TRP in CD and $2.9 \mathrm{~g}$ in LPD), only a large reduction in the TRP content of the diet (i.e. $0.3 \mathrm{~g}$ TRP in the TRPdeficient diet) may lead to a depletion of the TRP pool in the brain.

The effects of the valine-deficient diet were unusual. In the substantia nigra the effect on the serotoninergic system was similar to that found with other amino acid deficiencies, being the reverse of that in the striatum (5-HT concentration decreased in the substantia nigra and increased in the striatum).

\section{Effect of some essential amino acid deficiencies in the dopaminergic system}

When the catecholamine levels were studied there was a notable increase in TYR concentration after the administration of each of the protein-deficient diets in both regions studied. This effect was produced by LPD and it was maintained in all amino acid deficiencies studied with the exception of the phenylalanine-deficient diet. The maximum 
increase was seen after leucine deficiency in both regions. This may have been due to leucine and phenylalanine at normal plasma concentrations occupying more than $50 \%$ of the transport sites (Smith et al. 1987). Therefore, the decrease in leucine could have led to an increase in phenylalanine, with a concomitant increase of TYR concentration. However, in no case was the increase in TYR accompanied by an increase in the synthesis of DA. This is in agreement with the knowledge that DA regulation is independent of TYR concentration (Murrin et al. 1976), contrary to 5-HT synthesis to tryptophan (Miller et al. 1976, 1977b). A similar effect was seen by Westerink \& De Vries (1991) after TYR administration directly to the striatum; that is, it was without effect on either formation of 3,4-dihydroxyphenylalanine (DOPA) or release of DA. At the same time, with deficiencies of isoleucine, leucine, or tryptophan the increase in TYR concentration was associated with a decrease in DA concentration in the substantia nigra. These findings, together with the changes observed in DOPAC concentration which was either unchanged or increased (Leu deficiency), led to an increase in the DOPAC:DA ratio ( $0 \cdot 13$ for LPD, and 0.20, 0.22 and 0.19 for isoleucine, leucine and tryptophan deficiencies respectively) suggesting an increase in metabolism inside the cell. This hypothesis is supported by the few changes seen in the 3-MT:DA ratio. 3-MT is thought to reflect the level of DA in the synaptic cleft, since its formation occurs entirely extraneuronally (Westerink \& Spaan, 1982; Altar et al. 1987). Therefore, the different amino acid deficiencies did not seem to change this extraneuronal metabolism in either of the regions studied, being more evident in the striatum. This consistency in the 3-MT:DA ratio in the striatum could be related to a maintenance of the DA release process. The decrease in HVA concentration in the striatum was similar to that seen in DA concentration, as shown by the HVA:DA ratio in the striatum, which tended to decrease when the different amino acid-deficient diets were compared with both $\mathrm{CD}$ and LPD. This means that the amino acid deficiencies studied induced a metabolic change in DA that can be summed up as: (1) reinforcement of the intraneuronal metabolism of DA but not of the release processes, and (2) facilitation of DOPAC removal from the brain across the probenecid-sensitive transport system which has been reported to be involved in this process (Elchisak et al. 1977; Grabowska-Andén et al. 1984).

The deficit of phenylalanine (TYR precursor) counteracted the TYR-increasing effect produced by LPD in both structures. In the substantia nigra it produced a decrease in DA and DOPAC concentrations, which suggests a decrease in the rate of DA synthesis. This effect cannot be explained in the same way as the TRP effect on serotonin, since it is known that there is not a significant relationship between variation in TYR concentration and DA synthesis and metabolism, compared with that between TRP and 5-HT (Miller et al. 1976, $1977 b)$.

The deficit of valine, similar to that of serotonin, produced opposite changes in DA levels in the regions studied (substantia nigra and striatum). A similar finding has been reported previously under normal conditions in these regions (Cheramy et al. 1981). DA released into the substantia nigra seems to have a physiological role, as it inhibits the activity of dopaminergic cells by acting on dopaminergic autoreceptors (Nieoullon et al. 1977). The contrasting changes in DA and 5-HT levels in both regions could demonstrate an interaction between the two amines; it has been reported that DA release from dendrites in the substantia nigra influences the release of $5-\mathrm{HT}$ in various structures of the basal ganglia (Hery et al. 1980).

All these findings support the fact that neurotransmitters are affected by some amino acid-deficient diets. TRP, as expected, had a marked effect on serotonin levels. In addition, there is evidence that suggests amino acid deficiency increases susceptibility, particularly of the substantia nigra, to damage. The concentration of DA was also affected. This could be interesting in pathological conditions, some of which may be produced by environmental 
toxins (Donaldson et al. 1982; Tipton, 1988), where there is also a decrease in the concentration of this neurotransmitter in these regions.

This work was supported by Grant PB87-0929.

\section{REFERENCES}

Altar, C. A., Marien, M. R. \& Marsall, J. F. (1987). Time course of adaptations in dopamine synthesis, metabolism, and release following nigrostriatal lesions: implications for behavioral recovery from brain injury. Journal of Neurochemistry 48, 390399.

Bobillier, P., Seguin, S., Petitjean, F, Salvert, D., Touret, M. \& Jouvet, M. (1976). The raphe nuclei of the cat brain stem: a topographical atlas of their efferent projections as revealed by autoradiography. Brain Research 113, $449-486$.

Carlsson, A. \& Lindquist, M. (1978). Dependence of 5-HT and catecholamine synthesis on concentrations of precursor amino acids in rat brain. Naunyn Schmiedeberg's Archives of Pharmacology 303, 157-164.

Cheramy, A., Leviel, V.\& Glowinski, J. (1981). Dendritic release of dopamine in the substantia nigra. Nature 289. $537-542$.

Cohen, G. (1983). The pathobiology of Parkinson's disease : biochemical aspects of dopamine neuron senescence. Journal of Neural Transmission 19, Suppl., 89-103.

Donaldson, J., McGreggor, D. \& La Bella, F. (1982). Manganese neurotoxicity: a model for free radical mediated neurodegeneration? Canadian Journal of Pharmacology 107, 1398-1405.

During, M. J., Acworth I. N. \& Wurtman R. J. (1989). Dopamine release in rat striatum : physiological coupling to tyrosine supply. Journat of Neurochemistry 52, 1449-1454.

Elchisak, M. A., Maas, J. W. \& Roth, R. H. (1977). Dihydroxyphenylacetic acid conjugate: natural occurrenco and demonstration of probenecid-induced accumulation in rat striatum, olfactory tubercles and frontal cortex. European Journal of Pharmacology 41, 369-378.

Enna, S. J., Stern. L. Z., Wastek, G. J. \& Yamamura, H. I. (1977). Neurobiology and pharmacology of Huntington's disease. Life Sciences $20,205-212$.

Fernstrom, J. D. \& Wurtman, R. J. (1971). Brain serotonin content: physiological dependence on plasma tryptophan levels. Science 173, 149-152.

Fornstedt, B., Pileblad, E. \& Carlsson, A. (1990). In vivo autooxidation of dopamine in guinea-pig striatum increases with age. Journal of Neurochemistry 55, 655-659.

Fuxe K. (1965). Evidence of the existence of monoamines in the central nervous system. IV. Distribution of monoamine nerve terminals in the central nervous system. Acta Physiological Scandinavica 247, Suppl., 37-85.

Gerson, S. \& Baldessarini, R. J. (1974). Nonspecific actions of dihydroxylated tryptamines in the central nervous system of the rat. Journal of Pharmacy and Pharmacology 26, 71-73.

Gomez-Reino, J., Abaitua, I, Diez Rojas, F. \& Campos, R. (1983). Estudio prospectivo del efecto de los corticoides y los scavenger de radicales libres en el tratamiento del S.T.E. (Prospective study on the effect of corticoids and free-radical scavengers in the therapy of the Spanish Toxic Syndrome. Programa de CSIC para el estudio del sindrome tóxico 1, 619-623.

Grabowska-Andén, M., Andén, N.-E., Barany, E. \& Magnusson, A. (1984). Organic acid transport to the blood from the corpus striatum, the thalamus and the cerebellum of the rat. Acta Pharmacologica et Toxicologica 54, 177-182.

Hery, F., Soubrie, P., Bourgoin, S., Motastruc, J. L., Artaud, F. \& Glowinski, J. (1980). Dopamine released from dendrites in the substantia nigra controls the nigral and striatal release of serotonin. Brain Research 193 , 143-151.

Hirsch, E. C., Graybiel, A. M. \& Agid, Y. A. (1988). Melanized dopaminergic neurons are differentially susceptible to degeneration in Parkinson's disease. Nature 334, 345-348.

Hothersall, J. S., Greenboum, A. L. \& McLean, P. (1982). The functional significance of the pentose phosphate pathway in sinaptosomes: protection against peroxidative damage by catecholamines and oxidants. Journal of Neurochemistry 39, 1325-1332.

Mann, D. M. A., Yates, P. O. \& Hawkes, J. (1982). The noradrenergic system in Alzheimer and multi-infarct dementias. Journal of Neurology, Neurosurgery and Psychiatry 45, 113-119.

Marsden, C. D. (1982). Neurotransmitters and disease; basal ganglia disease. Lancet ii, 1141-1147.

Miller, M., Leahy, J. P., McConville, F., Morgane, P. J. \& Resnick, O. (1977 a). Effects of developmental protein malnutrition on tryptophan utilization in brain and peripheral tissues. Brain Research Bulletin 2, 347-353.

Miller, M., Leahy, J. P., Stern, W. C., Morgane, P. J. \& Resnick, O. (1977b). Tryptophan availability: relation to elevated brain serotonin in developmentally protein-malnourished rats. Experimental Neurology 57, $142-152$.

Miller, M., Resnick, O., Leahy, J. P. \& Morgane, P. J. (1976). Brain and peripheral tryptophan availability in protein malnourished rats. Society Neuroscience Abstracts 2, 586.

Murrin, L. C., Morgenroth, V. H. \& Roth, R. H. (1976). Dopaminergic neuron effects of electrical stimulation on tyrosine hydroxylase. Molecular Pharmacology 12, 1070-108I. 
National Academy of Sciences (1978). Nutrient Requirements of Laboratory Animals. 3rd ed. Washington, DC: National Academy of Sciences.

Nieoullon, A., Cheramy, A. \& Glowinski, J. (1977). Release of dopamine in vivo from cat substantia nigra. Nature 266, 375-377.

Olsen, L. \& Seiger, A. (1972). Early prenatal ontogeny of central monoamine neurons in the rat: fluorescence histochemical observations. Zeitschrift für Anatomie und Entwicklungsgeschichte 137, 301316.

Pardridge, W. M. (1983). Brain metabolism: a perspective from the blood-brain barrier. Physiological Review 63 , 1481-1535.

Penney, J. B. \& Young, A. B. (1982). Quantitative autoradiography of neurotransmitter receptors in Huntington's disease. Neurology 32, $1391-1395$.

Reed, D. J., Brodie, A. E \& Meredith, N. J. (1983). Cellular heterogeneity in the status and functions of cysteine and glutathione. In Functions of Glutathione: Biochemical, Physiological, Toxicological \& Clinical Aspects, vol. 1, pp. 39-49 [A. Larsson, A. Holmgren, S. Orrenius and B. Mannervick, editors] New York: Raven Press.

Scheel-Krüger, J. (1986). Dopamine-GABA interactions: evidence that GABA transmits, modulates and mediates dopaminergic functions in the basal ganglia and the limbic system. Acta Neurologica Scandinavica 107, Suppl., $1-54$.

Schoefield, J. D. \& Booth, M. R. (1983). Wheat proteins and their technological significance. In Developments in Food Proteins, vol. 2, pp. 1-65 [B. F. J. Hudson, editor]. London: Applied Science Publishers.

Sies, H., Brigelius, R. \& Akerboom, T. P. M. (1983). Intrahepatic glutathione status. In Functions of Glutathione: Biochemical, Physiological, Toxicological \& Clinical Aspects, vol. I [A. Larsson, A. Holmgren, S. Orrenius and B. Mannervick, editors]. New York: Raven Press.

Smith, Q. R., Momma, S., Aoyagi, M. \& Rapaport, S. I. (1987). Kinetics of neutral aminoacid transport across the blood-brain barrier. Journal of Neurochemistry 49, 1651-1658.

Stachowiak, M. K., Stricker, E. M., Jacoby, J. H. \& Zigmond, M. J. (1986). Increased tryptophan hydroxylase activity in serotoninergic nerve terminals spared by 5,7-dihydroxytryptamine. Biochemical Pharmacology 35, [24]-1248.

Stern, W. C., Miller, M., Forbes, W. B., Leahy, J. P., Morgane, P. J. \& Resnick, O. (1976). Effects of protein malnutrition during development on protein synthesis in brain and peripheral tissues. Brain Research Bulletin 1, 27-31.

Tipton, K. F. (1988). Toxins and Parkinson's disease. Journal of the Irish Colleges of Physicians and Surgeons 17, $100-104$.

Ungerstedt, U. (1971). Stereotaxic mapping of the monoamine pathways in the rat brain. Acta Physiologica Scandinavica 367, Suppl., 1-48.

Van der Kooi, D. (1979). The organization of the thalamic, nigral and raphe cells projecting to the medial versus lateral caudate-putamen. A fluorescent retrograde double labelling study. Brain Research 169, 381-387.

Venero, J. L., Santiago, M., Machado, A. \& Cano, J. (1989). Determination of monoamines and both forms of monoamine oxidase in the rat's substantia nigra during postnatai development. Life Sciences $\mathbf{4 5}, 12771283$.

West, C. D. \& Kemper, T. L. (1976). The effect of a low protein diet on the anatomical development of the rat brain. Brain Research 107, 221-237.

Westerink, B. H. C. \& De Vries, J. B. (1991). Effect of precursor loading on the synthesis rate and release of dopamine and serotonin in the striatum : a microdialysis study in conscious rats. Journal of Neurochemistry 56, $228-233$

Westerink, B. H. C. \& Spaan, S. J. (1982). On the significance of endogenous 3-methoxytyramine for the effects of centrally acting drugs on dopamine release in rat brain. Journal of Neurochemistry 38, 680686.

Winick, M. (1989). Malnutrition and brain development. Journal of Pediatrics 74, 667-679.

Winick, M. \& Rosso, P. (1973). Effects of malnutrition on brain development. In Biology of Brain Dysfunction, vol. I, pp. 301-307 [G. E. Gaull, editor]. New York: Plenum Press. 